Materials and Methods We present data from the ENESTnd study. In ENESTnd, a phase 3, multicentre, open-label, randomised study, patients treated with nilotinib demonstrated higher and faster rates of major molecular response (MMR), more profound molecular response (MR), and complete cytogenetic responses (CCyR) compared with imatinib by 12 and 24 months. 282 adult patients were randomly assigned to receive nilotinib $300 \mathrm{mg}$ twice daily, 281 to receive nilotinib $400 \mathrm{mg}$ twice daily and 283 to receive imatinib. Patients were eligible if they had been diagnosed with chronic phase, Philadelphia chromosome-positive CML within the previous 6 months.

Results By 24 months after the start of treatment, significantly more patients had a MMR with nilotinib than with imatinib (201 with nilotinib $300 \mathrm{mg}$ twice daily, 187 with nilotinib $400 \mathrm{mg}$ twice daily and 124 with imatinib; $p<0.0001$ for both comparisons). Significantly more patients in the nilotinib groups achieved a complete molecular response at any time than did those in the imatinib group (74 with nilotinib $300 \mathrm{mg}$ twice daily, 59 with nilotinib $400 \mathrm{mg}$ twice daily and 29 with imatinib; $p<0.0001$ for nilotinib $300 \mathrm{mg}$ twice daily vs. imatinib, $p=0.0004$ for nilotinib $400 \mathrm{mg}$ twice daily vs. imatinib).

Conclusions Nilotinib continues to demonstrate superiority vs. imatinib with faster and more profound molecular responses. These results support nilotinib as a first-line treatment option for patients with newly diagnosed Philadelphia chromosome-positive and chronic myeloid leukaemia.

No conflict of interest.

\section{PHC-017 PHARMACOGENETIC STUDY ABOUT INFLUENCE OF A POLYMORPHISM IN GENE TRAILR1 IN RESPONSE TO INLFIXIMAB IN PATIENTS WITH CROHN'S DISEASE (CD)}

doi:10.1136/ejhpharm-2013-000276.362

'M de Andrés Bautista, 'D Lacruz Guzmán, ${ }^{2} V$ Dominguez Leñero, 'P Pérez Cañadas, 'M Martinez Penella, 'H Garcia Lagunar, 'E Ferris Villanueva, 'R Guerrero Bautista. ${ }^{1}$ Hospital Universitario Santa Lucia, Farmacia, Murcia, Spain; ${ }^{2 H o s p i t a l ~ P e r p e t u o ~}$ Socorro, Farmacia, Murcia, Spain

Background Anti-TNF drugs show high inter-individual variability in efficacy and toxicity.

Currently there are no genetic, biochemical or environmental markers to predict response to treatment.

Purpose To assess the influence of gene polymorphism rs2230229 TRAILR1 as a genetic marker in response to treatment with infliximab in patients diagnosed with Crohn's disease (CD). Will it enable us to predict response and improve the effectiveness of the drug?

Materials and Methods Prospective observational study of all patients diagnosed with CD treated with infliximab at our hospital. The assessment of response to infliximab was performed using as criteria of clinical response a decreased questionnaire score CDAI (Crohn Disease Activity Index) at the 4th dose. Subsequently patients were considered to have responded if their CDAI decreased by 70 points or more with respect the baseline and at least $25 \%$ on the total score and clinical remission was achieved by a CDAI of less than 150 points. Biological response criteria were defined such as patient responders, partial responders or non-responders according to variation in levels of C-reactive protein (CRP) with regard to baseline at 3, 6 and 12 months. To detect polymorphism KASPAR probes were used in a PCR-based allele-specific competitive FRET technology using a computer and a real time PCR of Aplied Biosystems $7500 \mathrm{~F}$ in $96-$-well plate. All patients included in the study received a starting dose of infliximab $5 \mathrm{mg} / \mathrm{kg}$ at 0,2 and 6 weeks after the start and then a maintenance dose every 8 weeks. Statistical analyses were performed with Epidat 3.1 and the level of significance was indicated by a $p$ value of less than 0.05 .
Results The study included a total of 40 patients. The mean age of the patients was $38.66 \pm 13.98$ years and $61.1 \%$ were female. The distribution for genotypes was $81.6 \% \mathrm{AA}, 15.8 \% \mathrm{GA}$ and $2.6 \% \mathrm{GG}$. Significant correlation wasn't found between genotypes or alleles of this polymorphism and clinical response to infliximab. Instead, statistically significant differences were shown for approximately 6 months of treatment when comparing patients with genotypes GG and GA/AA and a positive response $(p=0.047)$ when considering the biological response. Similarly patients with a $G$ allele had a more frequent negative response than those with the $\mathrm{A}$ allele $(p=0.043)$. On the other hand, significant correlation was found between patients carrying the A allele and the positive response, at 3,6 and 12 months based on biological response distribution.

Conclusions The results of our study show an association of this polymorphism with response to infliximab. Worst response rates are observed in patients carrying allele $G$ diagnosed with $C D$. We need more studies on this polymorphism and with a larger sample size to confirm these findings.

No conflict of interest.

\section{PHC-018 PHARMACOGENETIC STUDY AS A PREDICTOR OF EFFICACY AND TOXICITY IN PATIENTS WITH ADVANCED RENAL CELL CARCINOMA TREATED WITH SUNITINIB}

doi:10.1136/ejhpharm-2013-000276.363

II Cañamares-Orbis, ${ }^{2} \mathrm{JM}$ Sepúlveda-Sánchez, ${ }^{3} \mathrm{C}$ Rodríguez-Antona, ${ }^{2} \mathrm{CA}$ Farfan, ${ }^{1} \mathrm{C}$ García-Muñoz, 'S Cortijo-Cascajares, 'M Goyache-Goñi, "I Escribano-Valenciano. ${ }^{1}$ University Hospital 12 Octubre, Pharmacy, Madrid, Spain; ${ }^{2}$ University Hospital 12 Octubre, Medical Oncology, Madrid, Spain; ${ }^{3}$ Spanish National Cancer Research Centre, CNIO Cancer Center, Madrid, Spain

Background Sunitinib (SU) is an oral, small-molecule, multitargeted tyrosine kinase receptor inhibitor that is approved for the treatment of renal cell carcinoma (RCC). However, several patients either do not respond to treatment, or they do, but they experience significant toxicity.

Purpose To find genetic markers of toxicity and efficacy using a commercially available DNA microarray genotyping system.

Materials and Methods 25 patients with newly-diagnosed metastatic RCC were evaluated prospectively from January 2010 to May 2011. Patients received SU in repeated 6-week cycles of $50 \mathrm{mg} /$ day orally for 4 weeks, followed by 2 weeks off treatment. A total of 92 single nucleotide polymorphisms (SNPs) in 34 genes in the pharmacokinetic and pharmacodynamic pathways of drugs were analysed using the Drug inCode pharmacogenetic service. This test is performed from a saliva sample and uses a DNA microarray system. Polymorphisms in candidate genes, together with clinical characteristics, were tested by univariate analysis for association with the number of days of sunitinib treatment until the first reduction of dose, progression free survival (PFS) and overall survival (OS).

Results Patients with CYP1A2*1/*1, a low-metabolising genotype, needed dose reduction due to an increased risk of toxicity vs. * $1 \mathrm{~F} /{ }^{*} 1 \mathrm{~F}$ or $1 \mathrm{~F} / 1 \mathrm{~F} *$ (Median time to dose reduction: 2.33 months vs. not reached during study period; $p<0.006$ ). Patients with CYP2C19*1/*1, wild type genotype, had an increased risk of dose reductions due to toxicity versus other genotypes (Median time to dose reduction: 2.8 months vs. 9.73 months; $\mathrm{P}<0.021)$. No statistically significant associations were observed among drug metabolising genes and PFS or OS.

Val(158)Met Catechol-O-methyltransferase (COMT) gene polymorphisms have been associated with PFS and OS. We found that Met/Met carriers, low metabolising allele, had longer PFS and OS compared to those with Met/Val (PFS not reached vs. 15 months; OS not reached Vs17.2 months) and $\mathrm{Val} / \mathrm{Val}$ (PFS = 3.3 months; $\mathrm{OS}=4.4$ months $)$ phenotypes $(\mathrm{P}=0.005$ for $\mathrm{PFS}$ and $\mathrm{P}=0.003$ for OS). 
Conclusions This preliminary analysis suggests that CYP1A2 and CYP2C19 polymorphisms may be associated with toxicity in patients with RCC treated with sunitinib. Polymorphisms associated with toxicity and survival in this preliminary analysis are being validated in an independent cohort of 95 RCC patients treated with sunitinib.

No conflict of interest.

\section{PHC-019 PHARMACOGENETICS OF ANTIPLATELET AGENTS: TOWARDS PERSONALISED TREATMENT?}

\section{doi:10.1136/ejhpharm-2013-000276.364}

${ }^{1} \mathrm{C}$ Dávila Fajardo, 2J Sánchez-Ramos, 2P Toledo Frías, 'C García, 'C Gómez, 'C Marín, ${ }^{2} \mathrm{~A}$ Bautista, ${ }^{2} \mathrm{~F}$ Burillo, $1 \mathrm{~J}$ Cabeza Barrera. 'San Cecilio University Hospital, Hospital Pharmacy, Granada, Spain; ${ }^{2}$ San Cecilio University Hospital, Cardiology, Granada, Spain

Background Clopidogrel antiplatelet effects differ according to genotypes $\mathrm{ABCB} 1$ and $\mathrm{CYP} 2 \mathrm{C} 19$, establishing normal, intermediate and slow metabolizers. The intermediate and slow metabolizers and poor transporters are responsible for the poor response to the antiplatelet drug.

Purpose To determine the prevalence of CYP2C19 and ABCB1 genetic polymorphism in the normal Andalusian population (control) and compare it with other populations as a step to implement this determination in clinical practise.

Materials and Methods We genotyped 100 controls from the Andalusian DNA bank for CYP2C19 * 2 (rs4244285), CYP2C19 * 3 (rs4986893) and ABCB1 (rs1045642) using TaqMan probes and allelic discrimination technique. Statistical analysis for allelic and genotypic distributions was calculated by chi-squared test or Fisher's exact test, when necessary, using the Statcalc software packages.

Results Genotype frequencies CYP2C19 $\left({ }^{*} 2\right)$ in the Andalusian population: ${ }^{*} 1 /{ }^{*} 1$ : $73 \%,{ }^{*} 1 /{ }^{*} 2: 25 \%,{ }^{*} 2 /{ }^{*} 2: 2 \%$, and CYP2C19 ${ }^{*} 3$ : none; the same results as in HapMap (NW European ancestry) population. ABCB1: Andalusian population: CC 36\%, CT 44\%, TT 20\%; HapMap population CC $27 \%$, CT 50\%, TT 23\%. Allelic frequencies: NW European ancestry HapMap CYP2C19 * 2: G is $85 \%$ and A is 15\%, the same as our Andalusian control results. ABCB1: HapMap $\mathrm{C}$ allele frequency is $45 \%$ and the $\mathrm{T}$ is $55 \%$, and our frequencies are $57 \%$ C and $43 \%$ T. Having made the genotype study, $59 \%$ of the controls were sensitive to clopidogrel and $41 \%$ resistant to it.

\section{Conclusions}

- Frequencies for CYP2C19*2 and *3 were similar to those reported in other studies. The frequencies for ABCB1 differed slightly

- It is necessary to perform this type of study in patients with acute coronary syndrome undergoing a percutaneous coronary intervention, to ensure effective treatment as it is documented that clopidogrel is not an effective drug in polymorphisms with allele CYP2C19 $* 2\left(* 1 /{ }^{*} 2\right.$ and $\left.{ }^{*} 2 /{ }^{*} 2\right)$ and/or ABCB1 TT.

No conflict of interest.

\section{PHC-020 PHARMACOKINETICALLY GUIDED DOSE ADJUSTMENT OF 5-FLUOROURACIL (5-FU) IN GASTROINTESTINAL CANCER PATIENTS}

doi:10.1136/ejhpharm-2013-000276.365

A Egüés, A Aldaz, M Marin, N Alzueta, L Zufia, A Bermejo. Clinica Universitaria de Navarra, Pharmacy, Pamplona, Spain

Background Appropriate dosing of chemotherapeutic drugs is critical to reducing mortality and increasing progression-free survival. 5-fluorouracil (5-FU) is a widely used chemotherapeutic drug in gastrointestinal cancer. The standard approach to dosing $5-\mathrm{FU}$ is based on body surface area (BSA). However, BSA does not account for many of the factors that are responsible for 5-FU clearance such as age, gender, genotype, disease state, drug-drug interactions, organ dysfunction and co-morbidities. Clinical evidence indicates a strong correlation between both toxicity and therapeutic efficacy and total 5-FU exposure expressed as area under the curve (AUC) concentration. This evidence make 5-FU a good candidate for pharmacokinetic (PK)-guided dosing.

Purpose To evaluate the role of therapeutic drug monitoring (TDM) of 5-FU in daily clinical oncology practise.

Materials and Methods Prospective study of adult patients diagnosed with gastrointestinal cancer treated with infusion schedule regimes based on high doses of 5-FU $\left(2.5-3.2 \mathrm{~g} / \mathrm{m}^{2}\right.$ in $24-46 \mathrm{~h}$ infusion) in a university hospital. All patients were included regardless of disease state or general clinical status. Individual pharmacokinetic parameters were calculated based on anthropometrics and history of 5-FU administration using the Bayesian software programme (USC*Pack). In the first cycle the dose was calculated using the BSA, and subsequent doses were adjusted to an optimal target AUC of 30-35 $\mathrm{mg} \cdot \mathrm{h} / \mathrm{L}$.

Results Fifty-four patients were included in the study. Male/ female ratio was $31 / 23$, and average age and weight were $60.9 \pm 12.8$ years and $72.2 \pm 16.9 \mathrm{Kg}$. Mean estimated pharmacokinetic parameters for volume of distribution and 5 -FU clearance were $0.49 \pm 0.08$ $\mathrm{L} / \mathrm{Kg}$ and $203 \pm 68.6 \mathrm{~L} / \mathrm{h}$, respectively. To achieve the target AUC of $30-35 \mathrm{mg} \cdot \mathrm{h} / \mathrm{L}$, the dose had to be increased in $33(86.8 \%)$ patients and adjusted downward in $5(13 \%)$. No adjustment was needed in 16 patients $(29.6 \%)$. When the estimate was based on BSA, 30 patients $(55.6 \%)$ had AUC $<25 \mathrm{mg} \cdot \mathrm{h} / \mathrm{L}$

Conclusions BSA-based 5-FU dosing approaches are limited when it comes to achieving optimal plasma levels in most patients. Pharmacokinetically guided dosing represents a better strategy to improve the efficacy and safety of 5-FU.

No conflict of interest.

\section{PHC-021 PHARMACOKINETICS OF PIPERACILLIN AND CIPROFLOXACIN IN CRITICALLY ILL PATIENTS UNDERGOING CONTINUOUS VENOVENOUS HAEMODIALYSIS (CVVHD) OR CONTINUOUS VENOVENOUS HAEMODIAFILTRATION (CVVHDF)}

doi:10.1136/ejhpharm-2013-000276.366

F Scheer, I Kraemer. University Medical Center, Pharmacy Department, Mainz, Germany

Background Critically ill patients on intensive care units are often suffering from sepsis and multiorgan failure causing a high mortality rate. In the presence of acute renal failure (ARF) survival can be improved by continuous renal replacement therapy (CRRT). However these procedures are known to be associated with underdosing of the antibiotic agents.

Purpose To investigate the efficacy and safety of antibiotic treatment, especially piperacillin/tazobactam and ciprofloxacin in patients undergoing CRRT.

Materials and Methods A total of 24 patients with ARF treated with CRRT were enrolled in the clinical trial. Plasma and dialysate concentrations of piperacillin/tazobactam and ciprofloxacin were measured in the steady state treatment phase. Serum concentrations of piperacillin and ciprofloxacin were analysed by a validated HPLC method. Optimum exposure to piperacillin is to be expected when serum concentrations are maintained 4-5 times higher than the minimal inhibitory concentration (MIC), i.e. above $64 \mathrm{mg} / \mathrm{l}$ Optimum exposure to ciprofloxacin is given when the ratio (AUIC) of the area under the curve (AUC) and MIC is $\geq 125 \mathrm{~h}$. In addition the Cmax/MIC ratio should amount to $\geq 10$.

Results For 10 of 21 patients treated with piperacillin/tazobactam 4/0.5g three times a day plasma concentrations lower than $64 \mathrm{mg} / 1$ 